\title{
XMILE - An Expert System for Maintenance Learning from Textual Reports
}

\author{
Eduardo Julião Máximo \\ Programa de Pós-Graduação em Informática \\ Aplicada \\ University of Fortaleza - UNIFOR \\ Fortaleza, Brazil \\ ejmaximo@gmail.com
}

\author{
Vládia Pinheiro \\ Programa de Pós-Graduação em Informática \\ Aplicada \\ University of Fortaleza - UNIFOR \\ Fortaleza, Brazil \\ vladiacelia@unifor.br
}

\begin{abstract}
Software incidents are normally described in natural language (like English or Portuguese languages), because the users become free to express themselves about the incident. In this paper, we propose XMILE - an eXpert MaIntenance LEarning system based on NLP (Natural Language Processing) and machine learning techniques, that is capable of inferring the main attributes (type of intervention, maintenance action, cause and faulty zone) from textual reports of incidents. The XMILE was used on a real set of reports of maintenance incidents performed on IT systems of a Brazilian automobile enterprise, with excellent results in terms of precision and recall metrics.
\end{abstract}

\section{INTRODUCTION}

Nowadays, the massive use of information systems and technologies (IT) requires a more specialized structure in IT support services and effective incident management. Incidents are some kind of unplanned interruption or reduction of the quality of an IT service, generating direct or indirect impact to the business. Initially, it is necessary that incidents be recorded or reported to a service center, generating software maintenance experiences databases. Several researches have proposed the reuse of experiences in Incident Management [1] and models such as the Information Technology Infrastructure Library (ITIL), contributing, for example, to the compliance with the rules of priority and time of attendance of the registered incidents, defined in Service Level Agreements (SLA).

The dissemination of Computerized Maintenance Management Systems (CMMS) has contributed to enriching Experiences Databases, which have been mainly used for traceability purpose. However, these bases could be processed in order to make explicit the "implicit knowledge", in order to improve the decisions related to the maintenance activity. However, its extraction can hardly be done manually. In [2], the authors propose an original Experience Feedback process dedicated to maintenance, allowing capitalizing on past activities by formalizing the domain knowledge and experiences using Conceptual Graphs (CGs) [3]. The basis of the Experience Feedback process is an ontology that mainly allows modeling the maintenance interventions according to the three main parts of an experience: (i) context, that describes the general situation in which the event has

DOI reference number: 10.18293/SEKE2018-197 occurred (i.e. service order, functional localization of equipment involved, failure, technician); (ii) analysis, that presents the cause(s) of the problem; (iii) solution, that describes the type of intervention and the actions that have been performed for solving this problem.

However, software incidents are normally described in natural language (like English or Portuguese languages), because the users become free to express themselves about the incident. On the other hand, textual reports are a non-structured knowledge source, making the process of extracting information about the incident more difficult. Because of this, traditional CMMS use the form of description of maintenance experiences by attribute-value, whereby structured fields need to be informed manually by users or IT support technicians, making the process very time consuming.

In this paper, we propose XMILE - an eXpert MaIntenance LEarning system based on NLP (Natural Language Processing) and machine learning techniques, that is capable of automatically extracting information about the maintenance from incidents textual reports, inferring their main attributes. According to [2], we define four main attributes: type of intervention, maintenance action, cause and faulty zone. These attributes are required in order to respond the following question, respectively: in which conditions?, what?, why?, and where? For example, for the incident report "Please check for TGA and $S G$ must be separate information and program assumes the same information", XMILE extracts: Type of intervention = "IT System"; Fault Zone ="System functionality" and Cause="System Failure".

A distinguishing feature of XMILE is the possibility of making explicit the information about maintenance cases (problems and solutions) described in natural language, for example, in the Portuguese language, in real time and without human intervention. The XMILE was used in a real set of reports on maintenance incidents performed on IT systems of a Brazilian automobile enterprise, with excellent results in terms of precision and recall metrics.

\section{BACKGROUND KNOWLEDGE}

Our work is inspired in [2], which proposes a Experience Feedback Process consisting of two main modules that are presented in the next subsections. 


\section{II.1 Experiences Database Structure}

The basis of a knowledge-oriented representation of the experiences is an ontological knowledge, that can be considered as a rudimentary ontology [4] and is essentially defined as a couple (TC, TR), representing respectively the hierarchy of concept types and the hierarchy of relation types. This ontology is a high level and generic knowledge on the domain, specifying the vocabulary of the maintenance domain and the semantics of this conceptual vocabulary [5]. Since maintenance is a matter of communication between operators, maintenance actors and experts of various fields, a specific attention has been drawn to ontologies, ensuring that information/knowledge exchanged by different actors is meaningful, and that all the stakeholders interpret it in the same way [6]. An ontology is defined in [7] as "a formal, explicit specification of a shared conceptualization". The "formal" aspect allows guaranteeing that the ontology is machine-readable [8]. The components of an ontology should thus allow formalizing the experience-knowledge in a specific domain.

TC has three main components of an experience: context, analysis and solution. In TC, the "context" part describes the general situation in which the event has occurred (i.e. Work Order (WO), functional localization of equipment involved, failure, technician); the " "analysis" part presents the cause(s) of the problem; finally, the "solution" describes the type of intervention and the actions that have been performed for solving this problem (i.e. selected maintenance activities). TR expresses the basic relations of generic ontologies that will be used here, like "temporal" relation (i.e. before, after, parallel), "spatial" relation (i.e. in, out), "logic", relation (e.g. implies), "usual" relation (i.e. object, agent, involve, etc.) [9], as well as other specific relations of the domain of study, such as "experience relation" (i.e. generates, requires) or 'element of'. These relations allow to link the different concepts types in the representation of an experience. An example is that a Context "require" an Analysis, which "generate" a Solution. In the Context we have that a WorkOrder has an "object" FunctionalLocalization and "concern" to a Technician. An Analysis is described by the concept Cause. Finally, the Solution graph defines that a TypeOfIntervention "concern" a MaintenanceAction.

\section{II.2 Rules Database Generation}

This module applies a data mining process on the Experience Databases in order to discover association rules. An association rule is formally defined as a relation between two attributes of experience $\mathrm{X}$ and $\mathrm{Y}$ (antecedent and consequent) contained in the Experience database, based on conditional probability $\mathrm{P}(\mathrm{X} \mid \mathrm{Y})$ and $\mathrm{P}(\mathrm{X} \cap \mathrm{Y})$. The association rules are selected according thresholds. After the association rules generation, the authors propose a semi-automatic process for assessment and interpretation of the rules.

\section{XMILE - A EXPERT SyStem FOR MAINTENANCE LEARNING FROM TEXTUAL REPORTS}

The reports of software incidents and maintenance results are normally described in natural language, by phone, e-mail, chat, or by tools for IT services monitoring, such as SpiceWorks ${ }^{1}$, OTRS (Open Ticket Request System) $)^{2}$, GLPI (Gestionnare Libre de Parc Informatique $)^{3}$, among others. As an IT service management needs the experiences databases in order to reuse the experiences and to improve the decisions related to the maintenance activity, we propose XMILE, an expert system based on NLP and machine learning techniques that automatically extracts the main attributes of textual incident and maintenance reports, generating knowledge in software maintenance. Initially, in this section, we define the ontology of the XMILE system and, after, we detail its architecture.

\section{III.1 XMILE Ontology}

Inspired on the generic model of an experience, defined by [2], we instantiate this model for IT maintenance experiences. An IT Experience consists of three parts: Context, Analysis and Solution. In the Context part, the new concepts are: (1) User - represents the user that report the incident or the user that usually operates the IT system, and is defined by the following attributes: (a) Admission Date, representing the date of the admission of the user in the company or function; and (b) Last Training Date, representing the date of last training that the user participated. These attributes aim to capture the user experience in the use of the system, as we believe that the user experience impacts on the number of incidents; (2) Text Report - represents the textual report of software incident or software maintenance.

We propose the following types of Fault Zone: (a) Equipment - any hardware device that, connected directly or indirectly to a computer, adds new functionality or whatever is required for a task; (b) Net access infrastructure (hardware, software and protocols, configuration) that enables LAN, WAN or Wi-Fi network connectivity; (c) System functionality - software or function of the IT system that performs the functional requirements of the users.

In the Analysis part, the causes of an IT Experience are: (a) Lack of Maintenance - indicating that there was failure to perform preventive maintenance on computers, printers, networks, and other devices; (b) Lack of Training - difficulty of the user to understand how the IT System work and how to reach its objectives; (c) IT System Failure - interruption of one or more tasks due to errors in IT system operating; (d) IT System Error - interruption of one or more tasks due to errors in IT system coding; (e) Lack of Permission - lack of permission granted to the user to produce or extract some information from the IT system; (f) Communication Problem - interruption in the provision or exchange of information on the internal network or with the Internet.

In the Solution part, the new concepts and instances are:

Type of Intervention - (a) Infrastructure - components and services (hardware and basic software) that provide the basis for sustaining all the information systems of an organization; (b) IT System - Automated or manual model of processes that use information technologies and

\footnotetext{
${ }^{1}$ https://www.spiceworks.com, accessed 03/14/2018

2 https://www.otrs.com, accessed 03/14/2018

${ }^{3} \mathrm{http}$ ://glpi-project.org, accessed 03/14/2018
} 
that are responsible for collecting and transmitting data that are useful for the development of products or services of companies, organizations and other projects; (c) Technical Support - Intellectual (knowledge), technological (hardware or software updates) and material, for the purpose of solving technical problems; (d) User Support - clarification of doubts, complaints, requests for services or support in solving problems.

Maintenance Action - (a) IT System update application of security patches, configurations, features, and other new or revised items that will change the current system; (b) IT System correction - adjustment in programs where you change the system default behavior; (c) IT Training onsite - acquisition of knowledge, skills and competences as a result of vocational training or teaching practical skills related to specific useful skills; (d) Infrastructure repair - installation, monitoring and updating of servers, printers and other devices in order to maintain the high availability and normal operation of the system.

\section{III.2 The XMILE System}

Figure 1 presents the architecture of the XMILE system with three components: Pre-processing, NLP and Machine Learning.

\section{Pre-Processing Component}

In the Pre-Processing component are performed the text mining and cleaning, data selection and data transformation. In order to prepare the data in an adequate format for the next NLP and Machine Learning components, we adopt here a data structure with the past experiences databases, based on the ontological knowledge (see Section III.1). This data structure is a triplet $\mathrm{D}=(\mathrm{O}, \mathrm{I}, \mathrm{R})$, in which $\mathrm{D}$ is the database, $\mathrm{O}$ is a set of objects or transactions (i.e. each maintenance experience), $\mathrm{I}$ is a set of attributes (i.e. concepts defined in the XMILE ontology) and $\mathrm{R} \subseteq \mathrm{O} \times \mathrm{I}$ is a binary relation between $\mathrm{O}$ and $\mathrm{I}$. Thus, each maintenance experience $\mathrm{O}$ in $\mathrm{D}$ is represented by a set of concepts in I related by binary relations in $\mathrm{R}$. It's important to note that a text of an incident report $\mathrm{t} \in \mathrm{T}$ is related with the concept Text Report in I. Next, we detail each phase:

- Text Cleaning and Mining - in this phase, the text is mined and cleaned by NLP processors like tokenizers and lemmatizers, then, stopwords and special characters are removed, and all letters are turned in uppercase. This phase aims at improving text quality, especially because the users and technicians write in an informal incomplete way. This is done by using NLP techniques that transform the tokens (word or expression) into their lemmas or canonical forms.

- Data Selection - in this phase, the values of each object in $\mathrm{O}$ is selected from the databases and associated to a concept in I. For example, given the incident "Please check for TGA and $S G$ must be separate information and program assumes the same information", reported by the userId $=9874$, which has data of admission $=09 / 12 / 2007$, and was trained last time $10 / 04 / 2014$. So, we will have the following concept and values: User.admissionDate = 09/12/2007; User.lastTrainingDate $=10 / 04 / 2014$
- Data Transformation - in this phase, the same data is transformed and normalized. For example, if in the original database, the date was in long format, we can transform in short format.

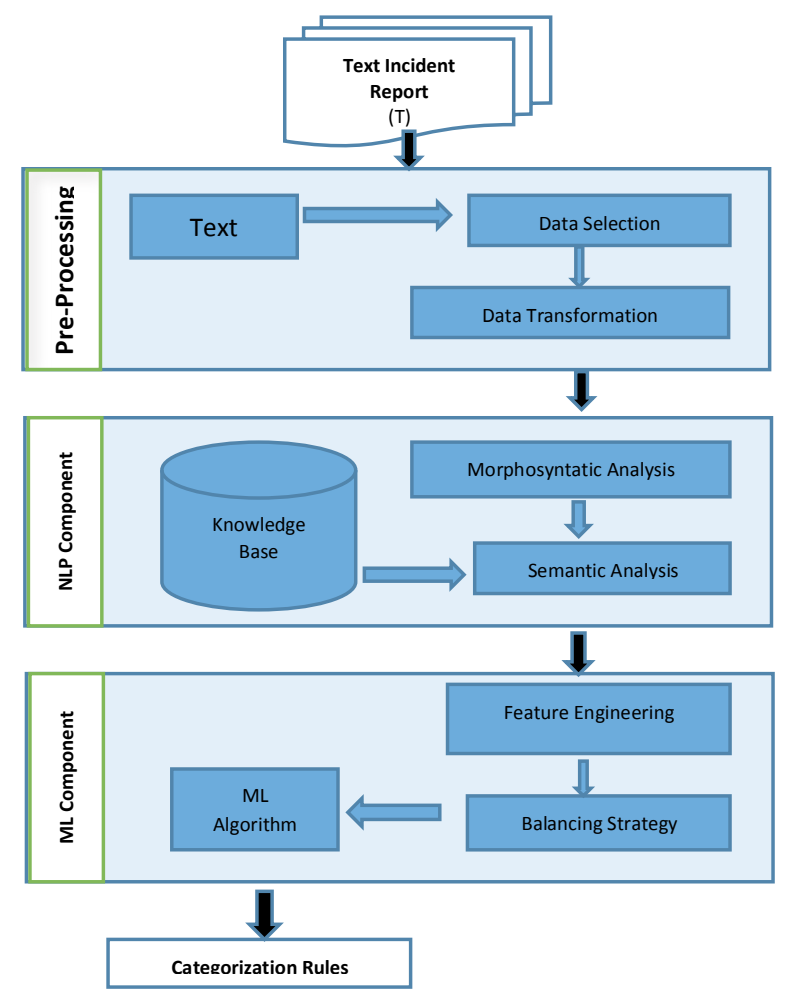

Figure 1. THE XMILE'S ARCHITECTURE.

\section{NLP Component}

- Morphologic Analysis - this component performs the morphological analysis or shallow parsing of the texts. Morphological analysis consists of the separation of sentences and grammatical classification (noun, verb, adjective, adverb, etc) and their modifiers of gender, number, time and verbal mode. Shallow parsing is applied to facilitate and normalize the search for word meaning (disregarding verbal, gender and number variations). In this component, we use a parser according to the language (e.g Freeling parser [10], for Portuguese Language, and Stanford Core NLP Toolkit [11], for English Language).

- Semantic Analysis - this component was developed to enrich the vocabulary with synonyms from a knowledge base like WordNet [12] or InferenceNet [13].

\section{Machine Learning Component}

This component is responsible for the calculation of the features (time of use of the equipment, time of the user in the organization, and quality of the internet/intranet connectivity), the balancing of the training and test sets, and the execution of Machine Learning algorithms, in order to learn rules for the categorization of attributes: Intervention type, Faulty zone, Cause and Maintenance Action. 


\section{EXPERIMENTAL EVALUATION}

In this experimental evaluation we want to verify the performance of XMILE in order to extract the information about the incident from textual report (in Natural Language).

In order to develop a golden standard, we selected 2819 textual incidents reports related to IT systems of a Brazilian company of automobile. Two IT technicians annotated each incident report with one of the Type of Intervention and Cause of the incident. In this work, we focus on these attributes because they represent the more important information to be extracted from incidents report, related to the Analysis and Solution of an Experience Database.

We defined two evaluation scenarios:

- $\quad$ SCENARIO 1 - we send to the Machine Learning algorithms only the bag of words of the textual reports (lemma of the relevant words)

- $\quad$ SCENARIO 2 - we send to the Machine Learning algorithms the bag of words and the following features: (i) time of use of the equipment; (ii) time (in years) of the user in the function; (iii) quality of connection of the Intranet or Internet.

In both scenarios, were executed three ML algorithms Random Forest, J48 and Naïve Bayes, with 10-cross fold validation and the balancing strategy SMOTE [14]. Table 1 and 2 present the results in terms of F1-Measure (harmonic average between precision and recall) for the attribute Type of Intervention and Cause, respectively.

TABLE 1. RESULtS FOR ATTRIBUTE TYPE OF INTERVENTION (F1MEASURE)

\begin{tabular}{|l|c|c|c|}
\hline & J48 & $\begin{array}{c}\text { NAIVE } \\
\text { BAYES }\end{array}$ & $\begin{array}{c}\text { RANDOM } \\
\text { FOREST }\end{array}$ \\
\hline SCENARIO 1 & 0.907 & 0.849 & $\mathbf{0 . 9 1 4}$ \\
\hline Infrastructure & $\mathbf{0 . 8 9 6}$ & 0.869 & $\mathbf{0 . 8 9 6}$ \\
\hline IT System & 0.858 & 0.780 & $\mathbf{0 . 8 7 8}$ \\
\hline Technical Support & 0.862 & 0.782 & $\mathbf{0 . 8 6 3}$ \\
\hline User Support & 0,881 & 0.820 & $\mathbf{0 . 8 8 8}$ \\
\hline Average & 0.907 & 0.811 & $\mathbf{0 . 9 1 2}$ \\
\hline SCENARIO 2 & $\mathbf{0 . 9 0 0}$ & 0.791 & $\mathbf{0 . 9 0 0}$ \\
\hline Infrastructure & 0.855 & 0.795 & $\mathbf{0 . 8 6 5}$ \\
\hline IT System & 0.855 & 0.785 & $\mathbf{0 . 8 6 2}$ \\
\hline Technical Support & 0.880 & 0.796 & $\mathbf{0 . 8 8 5}$ \\
\hline User Support &
\end{tabular}

TABLE 2. RESULTS FOR ATTRIBUTE CAUSE (F1-MEASURE)

\begin{tabular}{|l|c|c|c|}
\hline & $\mathbf{J 4 8}$ & $\begin{array}{c}\text { NAIVE } \\
\text { BAYES }\end{array}$ & $\begin{array}{c}\text { RANDOM } \\
\text { FOREST }\end{array}$ \\
\hline SCENARIO 1 & 0.723 & 0.670 & $\mathbf{0 . 7 7 2}$ \\
\hline Lack of Maintenance & 0.742 & 0.646 & $\mathbf{0 . 7 6 4}$ \\
\hline Lack of Training & 0.746 & 0.789 & $\mathbf{0 . 7 7 3}$ \\
\hline IT System Failure & $\mathbf{0 . 7 3 9}$ & 0.702 & 0.738 \\
\hline IT System Error & 0.803 & 0.701 & $\mathbf{0 . 8 2 1}$ \\
\hline Lack of Permission & 0.926 & 0.769 & $\mathbf{0 . 9 4 2}$ \\
\hline Communic Problem & 0.777 & 0.726 & $\mathbf{0 . 7 9 9}$ \\
\hline Average & 0.712 & 0.670 & $\mathbf{0 . 7 4 3}$ \\
\hline SCENARIO 2 & $\mathbf{0 . 7 5 0}$ & 0.684 & 0.740 \\
\hline Lack of Maintenance & $\mathbf{0 . 7 7 3}$ & 0.722 & 0.744 \\
\hline Lack of Training & 0.696 & 0.700 & $\mathbf{0 . 7 2 6}$ \\
\hline IT System Failure & 0.787 & 0.712 & $\mathbf{0 . 8 0 4}$ \\
\hline IT System Error & 0.916 & 0.809 & $\mathbf{0 . 9 1 7}$ \\
\hline Lack of Permission & 0.775 & 0.715 & $\mathbf{0 . 7 7 7}$ \\
\hline Communic Problem &
\end{tabular}

According to the results, we can observe that the best ML algorithm is the Random Forest with F1-measure = 0,888 and 0.799 , respectively. An interesting result is that the additional knowledge about the context (additional features about time in the function, equipment age, and quality of connectivity) does not influence the results.

\section{CONCLUSION}

In this paper, we propose XMILE - an expert system based on Natural Language Techniques that extract automatically the main attributes from a textual report that describes a software incident. We evaluate XMILE in a set of 2819 textual incidents reports related to IT systems of a Brazilian company of automobile and the expert system achieved $89 \%$ and $80 \%$ (f1-measure evaluation metric) for the main attributes - Type of Intervention and Cause of an incident. As future works, we intend to evolve XMILE to the other attributes of the ontology and to verify additional features that can improve its performance.

\section{REFERENCES}

[1] BEZERRA, G.; Pinheiro, V.; ALBUQUERQUE, A.Incident Management Optimization through the Reuse of Experiences and Natural Language Processing In: 9th International Conference on the Quality of Information and Communications Technology (QUATIC), 2014, 2014, Guimaraes.

[2] Ruiz, P Potes, FOGUEM, B Kamsu, GRABOT, BERNARD (2014). Generating knowledge in maintenance from Experience Feedback. Knowledge-Based Systems 68 (2014) 4-20.

[3] J. Sowa, Conceptual Structures: Information Processing in Mind and Machine, Addison-Wesley Publishing Company, Reading, MA, 1984.

[4] M. Chein, M.L. Mugnier, Graph-Based Knowledge Representation: Computational Foundations of Conceptual Graphs, Springer Publishing Company, Incorporated, 2008.

[5] F. Fürst, F. Trichet, Axiom-based ontology matching, Expert Syst. 26 (2) (2009) 218-246.

[6] M. Uschold, M. Grüninger, Ontologies: principles, methods and applications, Knowl. Eng. Rev. 11 (2) (1996) 93-136.

[7] T.R. Gruber, Toward principles for the design of ontologies used for knowledge sharing, Int. J. Hum.-Comp. Stud. 43 (5-6) (1995) 907-928.

[8] R. Studer, V.-R. Benjamins, D. Fensel, Knowledge engineering: principles and methods, Data Knowl. Eng. 25 (1-2) (1998) 161197.

[9] J.Breuker, A cognitive science perspective on knowledge acquisition, Int. J. Hum.-Comp. Stud. 71 (2) (2013) 177-183.

[10] Padró, Lluís e Evgeny Stanilovsky. 2012. Freeling 3.0: Towards wider multilinguality

[11] C.D. Manning, M. Surdeanum, J. Bauer, et al. The Stanford CoreNLP Natural Language Processing Toolkit. In Proceedings of 52nd Annual Meeting of the Association for Computational Linguistics: System Demonstrations, 2014.

[12] Miller, G. A. (1995). Wordnet: a lexical database for english. Communications of the ACM, 38(11):39-41.

[13] V. Pinheiro, T. Pequeno, V. Furtado and W. Franco. InferenceNet.Br: Expression of Inferentialist Semantic Content of the Portuguese Language. In: T.A.S. Pardo et al. (eds.): PROPOR 2010, LNAI 6001(90-99). Springer, Heidelberg, 2010.

[14] N.V. Chawla, K.W. Bowyer, L.O. Hall and W.P. Kegelmeyer. SMOTE: Synthetic Minority Over-sampling Technique. JAIR 16, 2002, pp. 321-357. 